\title{
Enhanced tumor immune surveillance through neutrophil reprogramming due to Tollip deficiency
}

\author{
Yao Zhang, ${ }^{1}$ Christina Lee, ${ }^{2}$ Shuo Geng, ${ }^{1}$ and Liwu $\mathrm{Li}^{1}$ \\ ${ }^{1}$ Department of Biological Sciences and 2Department of Translational Biology, Medicine, and Health, Virginia Tech, \\ Blacksburg, Virginia, USA.
}

\begin{abstract}
Although the importance of the tumor immune environment for the modulation of tumorigenesis and tumor regression is becoming increasingly clear, most of the research related to tumor-immune therapies has focused on adaptive immune cells, while the role and regulation of innate leukocytes such as neutrophils remains controversial and less defined. Here we observed that the selective deletion of Tollip, a key innate immune-cell modulator, led to enhanced tumor immune surveillance in a chemically induced colorectal cancer model. Tollip-deficient neutrophils significantly elevated T cell activation through enhanced expression of the costimulatory molecule CD80, and reduced expression of the inhibitory molecule PD-L1. Mechanistically, Tollip deficiency increased STAT5 and reduced STAT1, the transcription factors responsible for the expression of CD80 and PD-L1, respectively. Through adoptive transfer, we demonstrate that Tollip-deficient neutrophils, but not Tollip-deficient monocytes, are sufficient to drive enhanced tumor immune surveillance and reduced colorectal cancer burden in vivo. Our data reveal a strategy for the reprogramming of neutrophil functions conducive for the enhancement of the antitumor immune environment.
\end{abstract}

Authorship note: $\mathrm{YZ}$ and $\mathrm{CL}$ contributed equally to this work.

Conflict of interest: The authors have declared that no conflict of interest exists.

License: Copyright 2019, American Society for Clinical Investigation.

Submitted: June 20, 2018 Accepted: December 13, 2018 Published: January 24, 2019

Reference information: JCI Insight. 2019;4(2):e122939. https://doi.org/10.1172/jici. insight.122939.

\section{Introduction}

Decades of extensive studies have provided compelling evidence supporting a role for the immune system during the complex dynamics of tumor initiation, progression, and regression (1). Most notably, the roles of adaptive immune cells such as T cells in the recognition of tumor-specific antigens and coordination of antitumor functions have been well appreciated $(2,3)$. Recent advances suggest that innate immune cells including dendritic cells, monocytes, and neutrophils play vital roles in facilitating the antitumor functions of T cells, through affecting the expression and activities of immune checkpoint genes such as PD-L1 (4). Despite these exciting advancements, the roles and mechanisms of innate immune cells in the modulation of the tumor-immune environment remain less understood.

Among tumor-infiltrating innate immune cells, neutrophils are one of the major constituents (5). Solid tumor patients with poor prognosis tend to have expanded pools of tumor-associated neutrophils (5-7). Although the mechanisms are not well understood, neutrophils are known to exhibit complex and often opposing functions that either facilitate or prevent tumor initiation and growth (8). Differential expression of neutrophil cell surface molecules (e.g., PD-L1) as well as secretory mediators may contribute to the opposing functions of neutrophils in either augmenting or suppressing adaptive $\mathrm{T}$ cell activation. However, the molecular mechanisms underlying the differential activation of neutrophils are not well defined.

Tollip is an innate immunity signaling adaptor molecule expressed in myeloid cells (9). Initially recognized as an inhibitor for the TLR signaling pathway, recent studies suggest that Tollip may modulate cellular autophagy and other pathways in monocytes $(10,11)$. Its role in neutrophils, however, particularly in the setting of altered neutrophil function and the tumor immune environment, has not been studied.

In this study, we aimed to test the hypothesis that Tollip may contribute to the differential activation of neutrophils and that Tollip-deficient neutrophils may alter the colorectal cancer (CRC) immune environment. Despite improved care, CRC remains the third most commonly diagnosed cancer and the fourth leading cause of cancer mortality worldwide $(12,13)$. Our current study focused on testing the potential feasibility of intervening in the pathogenesis of CRC in an animal model with reprogrammed neutrophils. We tested the CRC tumor burden, immune environment, and neutrophil function of WT 
and Tollip-deficient mice challenged with azoxymethane-dextran sulfate sodium salt (AOM-DSS). We also performed adoptive transfer studies to specifically examine the role of Tollip-deficient neutrophils as well as monocytes in modulating the tumor immune environment and CRC formation. We observed that Tollip-deficient mice have reduced CRC burden and enhanced neutrophil-mediated $\mathrm{T}$ cell activation. Tollip-deficient neutrophils have elevated levels of costimulatory molecule CD80 and reduced levels of coinhibitory PD-L1, due to the induction of STAT5 and reduction of STAT1. We demonstrated that the transfusion of Tollip ${ }^{-/-}$neutrophils instead of Tollip ${ }^{-/}$monocytes into WT mice challenged with AOM-DSS can lead to reduced colorectal tumor burden. Our study reveals Tollip as a molecular checkpoint that governs the decision-making processes of neutrophils in shaping the CRC tumor immune environment.

\section{Results}

Tollip deficiency reduces colitis-associated tumorigenesis. In a previous study of acute DSS-induced colitis, we observed that Tollip ${ }^{-/-}$mice exhibit more severe acute colitis as compared with WT mice, due to elevated leukocyte infiltration and inflammation in the gut tissue (14). In the current study, we tested the severity of colorectal tumorigenesis in Tollip ${ }^{-/}$mice with the AOM-DSS model, which is a well-defined CRC model (15). Age- and gender-compatible mice (WT and Tollip ${ }^{--}$) were injected i.p. with a single dose of AOM (10 $\mathrm{mg} / \mathrm{kg}$ ) followed by 3 cycles of $2 \%$ DSS in the drinking water. A schematic of the protocol is provided in Figure 1A. While all mice survived the experimental periods (Supplemental Figure 1; supplemental material available online with this article; https://doi.org/10.1172/jci.insight.122939DS1), WT mice developed significant amounts of colon tumors throughout the distal as well as other segments of the colons (Figure 1, $\mathrm{B}$ and $\mathrm{C}$ ). In contrast, Tollip ${ }^{-/-}$mice exhibited a 50\% reduction in both microscopic and macroscopic polyps as compared with WT mice (Figure 1, B and C). We also monitored whole body health conditions including weight loss, stool consistency, and colorectal bleeding throughout the experimental course. Consistent with the acute colitis observations, we observed slightly more severe disease scores from Tollip ${ }^{-/}$mice following the initial cycle of DSS as compared with WT mice. In contrast, toward the end of the final DSS cycle, WT mice had much worse clinical scores including increased stool bleeding, consistent with more severe tumor burdens (Supplemental Figures 2, 3, and 4).

In addition to the general body outlook, we further performed a more focused histological assessment of the colon tissues. H\&E staining showed more severe colon inflammation and alterations of epithelial structure in WT mice as compared with Tollip ${ }^{-/}$mice (Figure 1D). In addition, to better define the cellular phenotype of tumorigenesis, we stained for Ki67, which serves as a well-recognized marker for hyperproliferative cells and tumorigenesis (16). WT mice with the AOM-DSS treatment exhibited pervasive Ki67 staining throughout the colon tissues (Figure 1E). In contrast, Tollip ${ }^{-/-}$mice similarly challenged with AOM-DSS demonstrated significantly reduced Ki67-positive cells in the colon, indicative of reduced tumorigenesis (Figure 1, E and F). $\beta$-Catenin is another well-studied independent marker for tumorigenesis (17). The cellular levels of $\beta$-catenin were also significantly decreased in the colon sections from Tollip ${ }^{-/}$ mice as compared with WT mice (Figure 1, G and H). Collectively, our data reveal that Tollip-deficient mice have reduced colon tumor formation when challenged with AOM-DSS.

Tollip deficiency enhances antitumor innate immune checkpoints and facilitates inflammation homeostasis. Since Tollip is recognized as a key modulator of innate immune cells, we asked whether enhanced antitumor defense in Tollip-deficient mice may be due to more effective anticancer checkpoints from innate immune cells. To test this, we first measured key innate checkpoint molecules expressed on neutrophils, such as PD-L1 and CD80. As shown in Figure 2A, splenic neutrophils from naive Tollip ${ }^{-/-}$ mice expressed significantly less PD-L1 and higher CD80 as compared with naive WT mice. This trend remained at the end of the AOM-DSS cycle (Figure 2A). The percentages of neutrophils within the blood and colon tissues remained similar among WT and Tollip ${ }^{-/}$mice before and after AOM-DSS challenge (Supplemental Figure 5).

Since colonic leukocytes are often recruited to the lamina propria (18), we next examined the levels of CD4 ${ }^{+}$ and $\mathrm{CD}^{+} \mathrm{T}$ cells present in this compartment in WT and Tollip ${ }^{-/-}$mice treated with AOM-DSS. At the end of the final DSS cycle, Tollip ${ }^{--}$mice had significantly higher amounts of both $\mathrm{CD}^{+}$and $\mathrm{CD} 8^{+} \mathrm{T}$ cells as compared with WT mice within the lamina propria where colonic leukocytes home into (Figure 2B). The numbers of $\mathrm{CD}^{+} \mathrm{T}$ cells in the spleen were also significantly higher in Tollip ${ }^{-/}$mice as compared with WT mice (Supplemental Figure 5). Correlated with elevated $\mathrm{T}$ cell populations, we observed elevated levels of IFN- $\gamma$ and IL-12 within colon tissues of Tollip ${ }^{-1-}$ mice as compared with WT mice following AOM-DSS challenge (Figure 2C). 
A

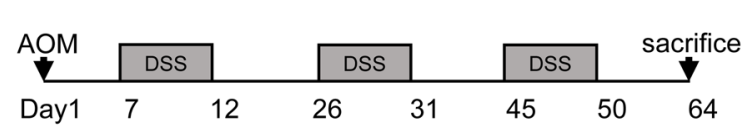

$\mathbf{B}$
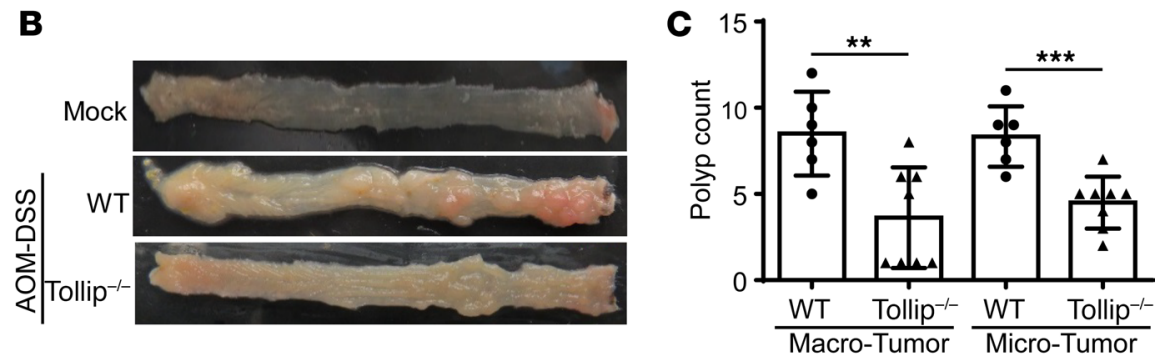

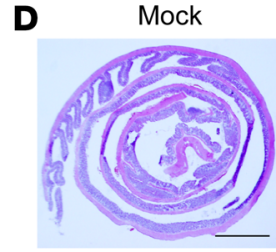

E

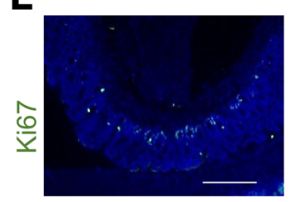

G

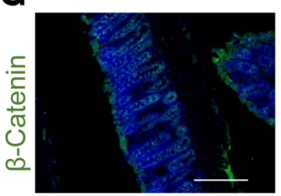

WT
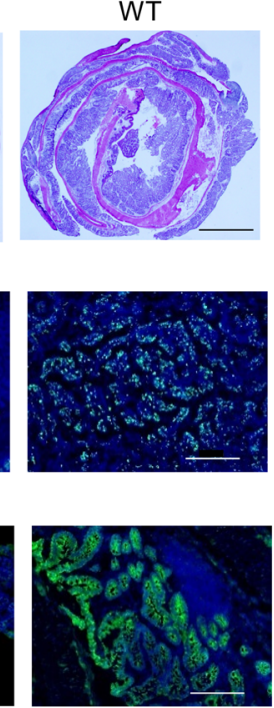
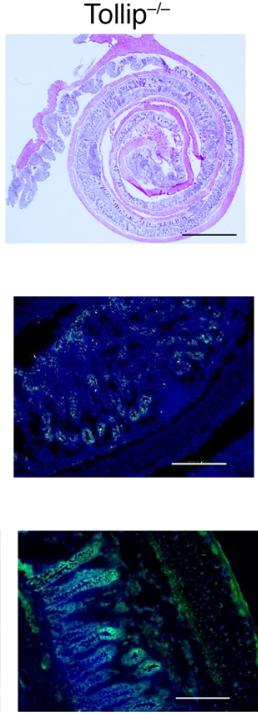

$\mathbf{F}$

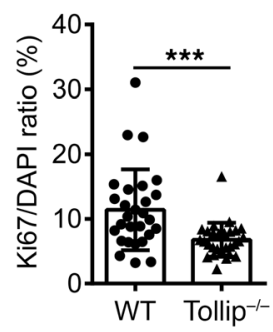

H

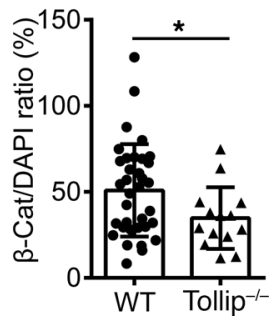

Figure 1. Tollip deficiency decreased tumorigenesis in the AOM-DSS mouse model of colorectal cancer. (A) Schematic protocol of AOM-DSS treatment. (B) Representative images of colons from WT and Tollip ${ }^{-1-}$ mice treated with AOM-DSS or naive mice. (C) Graphical representation of tumor burden in WT $(n=6)$ and Tollip ${ }^{-1-}(n=8)$ mice. Tumors with diameters greater than or equal to $2 \mathrm{~mm}$ were defined as "macro" tumors; those with diameters less than $2 \mathrm{~mm}$ were defined as "micro" tumors. (D) H\&E-stained sections of colon from WT or Tollip ${ }^{-/-}$mice treated with AOM-DSS or naive mice. Colons were collected in Swiss rolls at the end of the AOM-DSS regimen. Scale bars: $2 \mathrm{~mm}$. (E) Immunofluorescence analysis of Ki67 in colons of from WT or Tollip ${ }^{-1-}$ mice treated with AOM-DSS or naive mice. Scale bars: 200 $\mu \mathrm{m}$. (F) Quantitative analysis of Ki67 staining. (C) Immunofluorescence analysis of active $\beta$-catenin in colons from WT or Tollip ${ }^{-1-}$ mice treated with AOM-DSS or naive mice. Scale bars: $200 \mu \mathrm{m}$. (H) Quantitative analysis of active $\beta$-catenin staining. Statistical significance was determined by 1-way ANOVA followed by Tukey's post hoc comparisons (C) or Mann-Whitney $U$ test $(\mathbf{F}$ and $\mathbf{H}) .{ }^{*} P<0.05,{ }^{* *} P<0.01,{ }^{* * *} P<0.001$.

Consistent with reduced tumor burden in Tollip ${ }^{-/-}$mice, we observed reduced circulating inflammatory cytokine IL-1 $\beta$ in Tollip ${ }^{-/}$mice at the end of the AOM-DSS treatment regimen as compared with WT mice (Figure 2D). Circulating plasma levels of TGF- $\beta$ were significantly higher in Tollip ${ }^{-1}$ mice challenged with AOM-DSS as compared with WT mice (Figure 2D). Other inflammatory surface markers of circulating neutrophils such as CD14 and CCR5 were significantly lower in Tollip ${ }^{-/}$mice as compared with WT mice (Figure 2E). We also observed a similar reduction of CD14 on neutrophils collected from spleen and colon tissues of Tollip-deficient mice as compared with WT mice (Supplemental Figure 7). Our data suggest that Tollip deficiency may facilitate the resolution of chronic inflammation during AOM-DSS-induced colon tumorigenesis. We further surveyed other immune cells and did not observe any significant difference in the activation status of B cells, Treg cells, or monocytes in WT versus Tollip-deficient mice subjected to AOM-DSS challenge (Supplemental Figure 6).

Tollip mediates the immunosuppressive effects of neutrophils in suppressing $T$ cell proliferation. Based on our data showing that Tollip-deficient neutrophils exhibit enhanced CD80 and reduced PD-L1 levels, we further examined the role of Tollip-deficient neutrophils in modulating $\mathrm{T}$ cell function. It is well noted that 
A

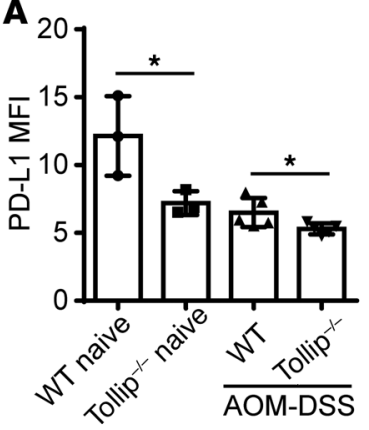

C

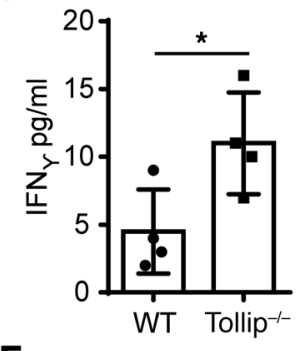

E

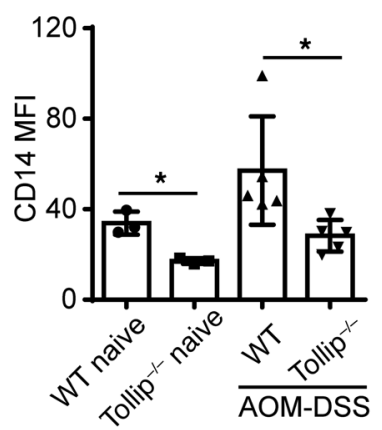

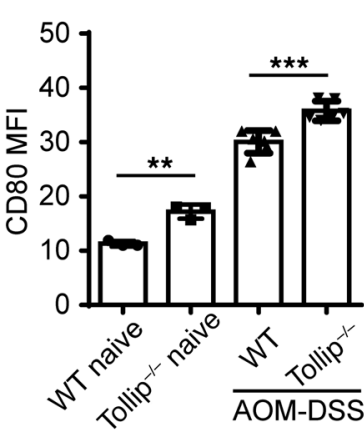

B
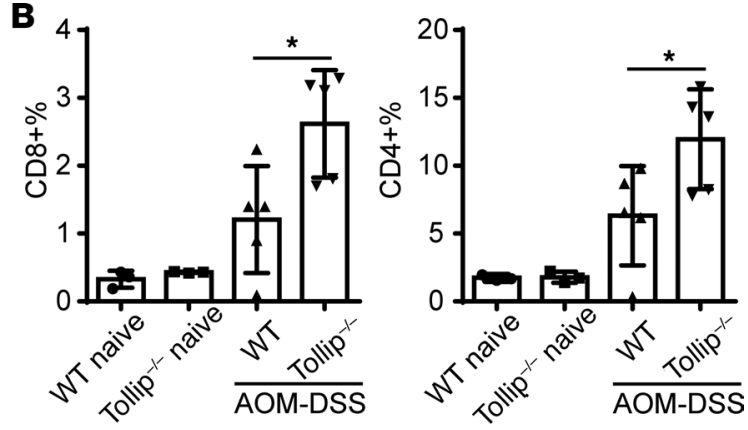

D
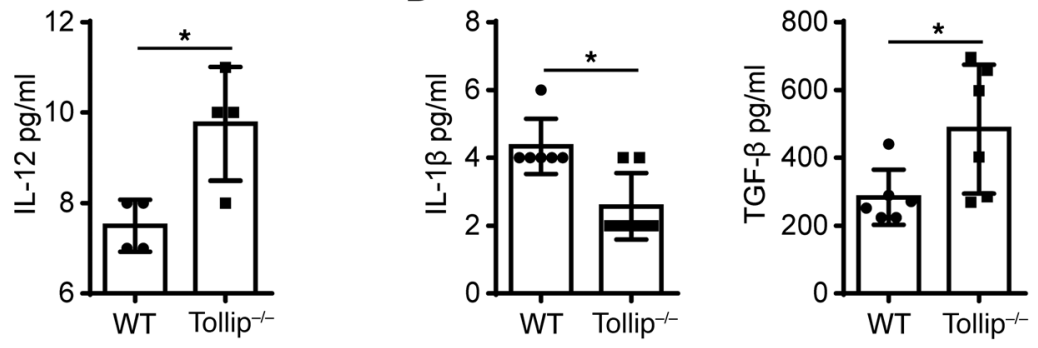

Figure 2. Tollip deficiency enhanced antitumor innate immune checkpoints. (A) PD-L1 and CD80 expression on the neutrophils in the spleens from WT or Tollip $^{-1-}$ mice with AOM-DSS treatment or naive mice. (B) Percentages of CD4+ and CD8 $8^{+}$cells in the colon lamina propria from WT or Tollip ${ }^{-1-}$ mice with AOMDSS treatment or naive mice. (C) Cytokine profiles of colons collected from WT or Tollip ${ }^{-/-}$mice treated with AOM-DSS. (D) Cytokine profiles of plasma collected from WT or Tollip ${ }^{-1-}$ mice treated with AOM-DSS. (E) CD14 and CCR5 expression on the surface of neutrophils in the blood. Statistical significance compared with WT in the same treatment conditions was determined by Student's $t$ test $(\mathbf{A}-\mathbf{C})$ or Mann-Whitney $U$ test $(\mathbf{D}$ and $\mathbf{E}) .{ }^{*} P<0.05,{ }^{* *} P<0.01,{ }^{* * *} P<0.001$.

tumor-associated neutrophils have immune-suppressive effects by suppressing $\mathrm{T}$ cell proliferation (5). To test the hypothesis that Tollip may facilitate the suppressive effects of neutrophils through modulating the expression of CD80 and PD-L1, we performed in vitro coculture studies. Increased production of granulocyte macrophage colony-stimulating factor (GM-CSF) has been observed in the mucosa of patients with inflammatory bowel disease and rodents subjected to experimental colitis. GM-CSF was also shown to promote the generation of myeloid-derived suppressor cells (19). We cultured bone marrow neutrophils from WT or Tollip $^{-1-}$ mice in GM-CSF overnight, and subsequently cocultured with CFSE-labeled allogeneic splenocytes in anti-CD3-coated plates. GM-CSF-primed neutrophils showed a typical immunosuppressive phenotype, as evident from reduced $\mathrm{T}$ cell proliferation upon the addition of neutrophils (Figure 3A). However, compared with WT neutrophils, Tollip ${ }^{-1}$ neutrophils had less immunosuppressive effects on the proliferation of both $\mathrm{CD}^{+}$and $\mathrm{CD} 8^{+} \mathrm{T}$ cells (Figure $3 \mathrm{~A}$ ). Consistent with in vivo results, Tollip ${ }^{--}$neutrophils had increased expression of CD80 and decreased PD-L1 expression (Figure 3B). To confirm the involvement of PD-L1 or CD80 on Tollip ${ }^{-1}$ neutrophils during the modulation of $\mathrm{T}$ cell proliferation, we applied blocking antibodies in the coculture assays. In the presence of anti-PD-L1 antibody, the suppressive effect of WT neutrophils on $\mathrm{T}$ cell proliferation was partially removed (Figure $3 \mathrm{C}$ ). In contrast, the application of anti-CD80 antibody blocked $\mathrm{T}$ cell proliferation (Figure 3D). These data suggest that Tollip ${ }^{-/}$neutrophils have reduced suppressive effects on T cell proliferation through an increase of CD80 and a decrease of PD-L1 expression. 
Tollip-deficient neutrophils enhance T cell survival and activation. Neutrophils may not only affect the proliferation and activation, but also the survival and activation of $\mathrm{T}$ cells. We further tested the effects of Tollip $^{-/}$neutrophils on $\mathrm{T}$ cell activation and survival in vitro using a coculture assay. For the $\mathrm{T}$ cell activation measurement, we tested the surface expression of CD62L, CD69, and CD107 $\alpha$ through flow cytometry, as well as secreted mediators such as IFN- $\gamma$ and granzyme B by ELISA. Following 1-day coculture of GM-CSF-primed neutrophils, both $\mathrm{CD}^{+}$and CD8 $\mathrm{T}^{+}$cells cultured with Tollip ${ }^{-/}$neutrophils exhibited downregulation of CD62L as compared with cells cocultured with WT neutrophils, indicating enhanced $\mathrm{T}$ cell activation (Figure 4A). The CD69 mean fluorescence intensity (MFI) was significantly increased in $\mathrm{CD}^{+} \mathrm{T}$ cells cocultured with Tollip-deficient neutrophils as compared with WT neutrophils, indicating elevated $\mathrm{CD}^{+} \mathrm{T}$ cell activation (Figure $4 \mathrm{~B}$ ). The populations of $\mathrm{CD} 107 \alpha$-expressing $\mathrm{CD}^{+} \mathrm{T}$ cells were also significantly elevated upon coculture with Tollip ${ }^{-/-}$neutrophils as compared with WT neutrophils, indicating elevated $\mathrm{CD}^{+} \mathrm{T}$ cell activation (Figure 4B). As measured by ELISA, the secreted levels of IFN- $\gamma$ and granzyme B from the cocultures with Tollip ${ }^{-1}$ neutrophils were significantly higher as compared with the cocultures with WT neutrophils (Figure 4C). Additionally, the application of anti-PD-L1 antibody facilitated the activation of $\mathrm{CD}^{+} \mathrm{T}$ cells and $\mathrm{CD} 8^{+} \mathrm{T}$ cells (Supplemental Figure 8). Anti-CD80 antibody also significantly reduced the activation of $\mathrm{CD}^{+} \mathrm{T}$ cells and $\mathrm{CD} 8^{+} \mathrm{T}$ cells (Supplemental Figure 8 ). To assess the effects of Tollip $^{-1-}$ neutrophils on $\mathrm{T}$ cell viability, we measured the viability of $\mathrm{T}$ cells following a 3-day coculture with either WT or Tollip ${ }^{-/-}$neutrophils using propidium iodide (PI) staining and flow cytometry. Both CD4 ${ }^{+}$and $\mathrm{CD}^{+} \mathrm{T}$ cells cocultured with Tollip $^{-/-}$neutrophils exhibited significantly higher survival rates as compared with T cells cocultured with WT neutrophils (Figure 4, D and E). Collectively, our data reveal that Tollip ${ }^{-/-}$ neutrophils enhance T cell activation as well as survival compared with WT neutrophils.

Tollip $^{-1-}$ neutrophils have elevated STAT5 activation and reduced STAT1 activation. We next examined the underlying molecular mechanisms responsible for the neutrophil reprogramming due to Tollip deficiency. Previous studies reported that STAT5 and STAT1 are differentially involved in the expression of CD80 and PD-L1, with STAT5 promoting the expression of CD80 (20) and STAT1 promoting the expression of PD-L1 $(21,22)$. Thus, we tested the activation of STAT1 and STAT5 as well as other key signaling molecules in WT and Tollip ${ }^{-/}$neutrophils. We observed that the phosphorylation levels of STAT1 were reduced in Tollip ${ }^{-1-}$ neutrophils as compared with WT neutrophils (Figure 5A). This is consistent with reduced PD-L1 expression in Tollip ${ }^{-/-}$neutrophils. In contrast, we detected an increased phosphorylation of STAT5 in Tollip ${ }^{-/}$neutrophils as compared with WT neutrophils, consistent with elevated expression of CD80 in Tollip ${ }^{-1-}$ neutrophils (Figure 5A). Correspondingly, we performed quantitative measurement of STAT1/STAT5 phosphorylation through flow cytometry. As shown in Figure 5B, Tollip ${ }^{-/-}$neutrophils had significantly elevated levels of p-STAT5 and reduced levels of p-STAT1, further corroborating our Western blot observations. Our data reveal that the dichotomy of elevated STAT5 activation and reduced STAT1 activation due to Tollip deficiency may underlie the polarized CD80/PD-L1 expression in Tollip-deficient neutrophils conducive for an effective T cell response toward tumor immune surveillance.

Adoptive transfer of Tollip ${ }^{-1-}$ neutrophils is sufficient to dampen colitis-associated tumor progression. Since neutrophils also play a vital role in host defense, experiments with neutrophil depletion would not be feasible with this particular long-term CRC model of AOM-DSS-induced gut damage and tumorigenesis. Alternatively, in order to directly test whether Tollip deficiency in neutrophils is responsible for antitumor efficacy in vivo, we performed an adoptive transfer experiment. Purified neutrophils from either WT or Tollip $^{-1-}$ mice were injected weekly via the i.v. route to WT mice subjected to AOM-DSS challenge, as described in the Methods section. We observed that WT mice receiving Tollip ${ }^{-1}$ neutrophils exhibited a marked reduction in the tumor load as compared with the WT mice receiving WT neutrophils (Figure $6, \mathrm{~A}$ and B). The overall body weight as well as colon length were similar among these groups at the end of the study (Supplemental Figure 9). However, only the colons from the mice transfused with Tollip ${ }^{-1-}$ neutrophils showed reduced inflammation (Figure 6C). Further, the colons from mice transfused with Tollip $^{-/}$neutrophils had reduced $\beta$-catenin and Ki67 staining when compared with those transfused with WT neutrophils (Figure 6D). Thus, transfusion of Tollip ${ }^{-/-}$neutrophils is sufficient to render protection against AOM-DSS-induced colon tumorigenesis.

To further examine this protective effect, we analyzed the levels and activation status of $\mathrm{CD} 4^{+}$and $\mathrm{CD}^{+} \mathrm{T}$ cells in mice transfused with WT or Tollip ${ }^{-/-}$neutrophils and subjected to the AOM-DSS challenge. As shown in Figure 6E, mice transfused with Tollip ${ }^{-/-}$neutrophils had increased numbers of splenic $\mathrm{CD}^{+}$and $\mathrm{CD}^{+} \mathrm{T}$ cells. Furthermore, $\mathrm{CD}^{+} \mathrm{T}$ cells in mice transfused with Tollip ${ }^{-/}$neutrophils 
A No
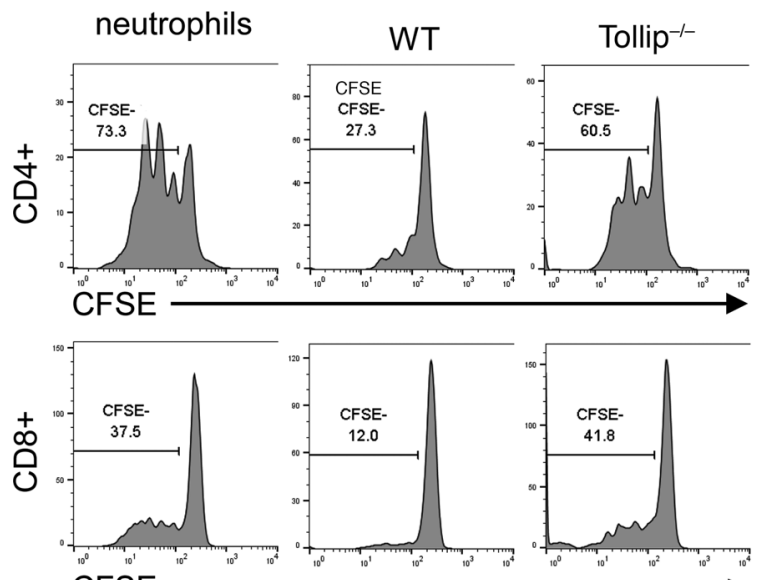

CFSE

$$
\text { c }
$$
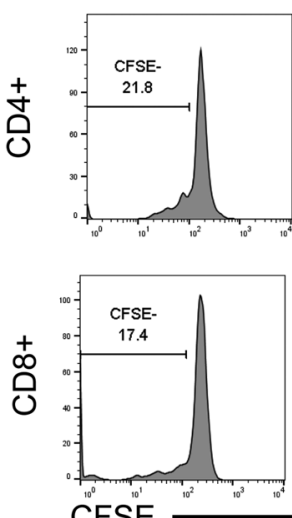

CFSE
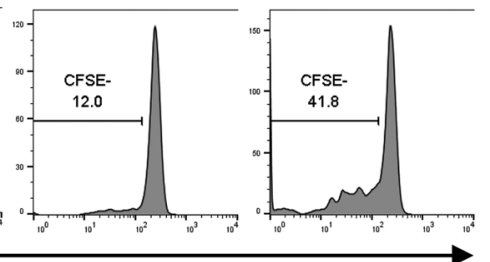

B
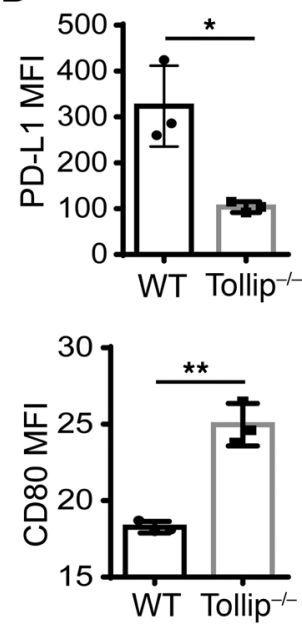

Figure 3. Tollip deficiency released the neutrophil suppression on T cell proliferation via PD-L1/CD80. (A) CFSE-labeled splenocytes were cocultured with GM-CSF-primed neutrophils in anti-CD3 antibody-coated plates for 72 hours. Representative results are shown. (B) PD-L1 and CD80 expression on GM-CSF-primed neutrophils. Statistical significance compared with WT in the same treatment conditions was determined by Welch's test. ${ }^{*} P<0.05$, ${ }^{*} P<0.01$. (C) In the presence of anti-PD-L1 antibody, CFSE-labeled splenocytes were cocultured with CM-CSF-primed WT neutrophils in anti-CD3 antibody-coated plates for 72 hours. (D) In the presence of anti-CD80 antibody, CFSE-labeled splenocytes were cocultured with GM-CSF-primed WT neutrophils in anti-CD3 antibody-coated plates for 72 hours.

demonstrated significantly elevated activation status as reflected in the higher percentage of $\mathrm{CD} 62 \mathrm{~L}^{10}$ as well as granzyme $\mathrm{B}^{+} \mathrm{CD} 8^{+} \mathrm{T}$ cells (Figure $6 \mathrm{~F}$ ). Our data reveal an enhanced in vivo antitumor immunity by transfusion of Tollip ${ }^{-/}$neutrophils.

Although our data suggest that reprogrammed neutrophils due to Tollip deficiency exhibit enhanced antitumor immune function in vitro and in vivo, we may not exclude the contribution of other innate leukocytes such as monocytes. To test whether Tollip-deficient monocytes may have similar effects, we performed an additional adoptive transfer study with WT and Tollip-deficient monocytes. Similar to the neutrophil study, monocytes from either WT or Tollip ${ }^{-/}$mice were i.v. injected weekly into WT mice subjected to AOMDSS challenge (Supplemental Figure 10). In contrast to the neutrophil transfusion, however, we observed no significant difference in colon tumor burden of recipient mice at the end of the experimental regimen (Supplemental Figure 10). However, we did observe that mice that received transfusion with Tollip-deficient monocytes exhibited longer colon length as compared with mice transfused with WT monocytes.

\section{Discussion}

Our current study reveals that, through Tollip deletion, neutrophils can be uniquely programmed to serve as highly effective immune modulators in the prevention and treatment of experimental CRC. 

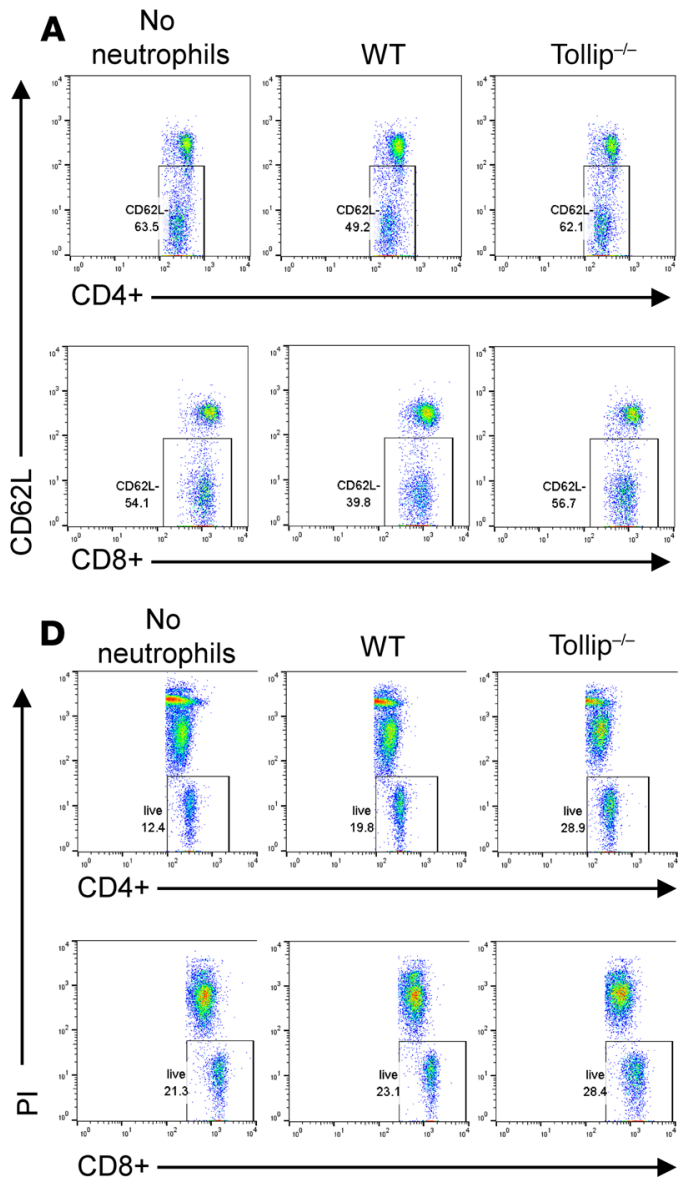

B
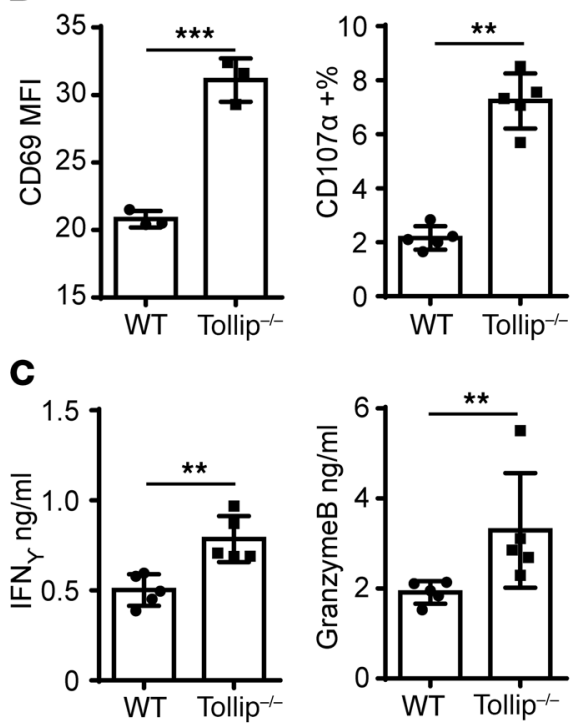

E

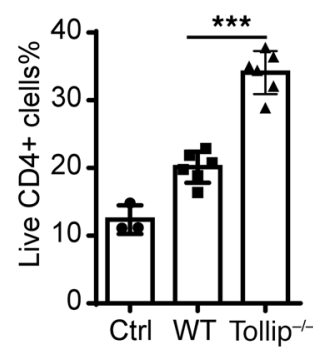

Figure 4. Tollip ${ }^{-/-}$neutrophils facilitated T cell activation and survival. (A) Splenocytes were cocultured with GM-CSFprimed neutrophils (WT or Tollip ${ }^{-1-}$ ) in anti-CD3 antibody-coated plates for 24 hours, and then CD62L levels on CD4+ or CD8 ${ }^{+} T$ cells were measured by flow cytometry. Representative results are shown. (B) After coculture, CD69 levels on CD4 ${ }^{+}$ cells and CD107a+ cells in CD8 ${ }^{+}$cells were analyzed. (C) Conditional medium from coculture was analyzed by ELISA. (D) Splenocytes were cocultured with GM-CSF-primed neutrophils (WT or Tollip ${ }^{-1}$ ) for 72 hours, before cell viabilities were tested. (E) Quantification analysis of the cell viabilities. Statistical significance compared with WT in the same treatment conditions was determined by Mann-Whitney $U$ test (B and $\mathbf{C}$ ) or Student's $t$ test $(\mathbf{E})$. ${ }^{*} P<0.05,{ }^{* *} P<0.01,{ }^{* * *} P<0.01$.

Several lines of data support this conclusion. First, we observed that Tollip-deficient mice have reduced colon tumor development when subjected to the AOM-DSS challenge. Second, we found that Tollip-deficient neutrophils are reprogrammed to be conducive for T cell proliferation, survival, and activation. Third, we demonstrated that the transfusion of Tollip-deficient neutrophils into WT mice is sufficient to alleviate AOM-DSS-induced colon tumor formation. Other supporting data include the lack of alterations in other leukocytes from Tollip-deficient mice and the failure of Tollip-deficient monocytes in conferring protection against CRC in mice challenged by AOM-DSS.

Our data provide a fresh perspective for the emerging and potentially important roles of neutrophils during the modulation of the tumor immune environment. Emerging basic and translational studies with experimental models and human cancer patients suggest complex repertoires of tumor-associated myeloid cells that may either promote or inhibit tumor progression (5, 23-26). Although the expanded pools of neutrophils within tumor tissues are very well-recognized common features closely correlated with aggravated tumor growth (5-7), it is not well understood how tumor-associated neutrophils are programmed at the molecular level to either facilitate or suppress tumorigenesis. Earlier studies led to the hypothesis that neutrophils may be differentially activated into either an N1 tumor-fighting state or an N2 tumor-promoting state (27-29). However, such categorization of neutrophils still lacks phenotypic and mechanistic clarity. Our data collected in this study extend these emerging studies and provide mechanistic insights into neutrophil reprogramming in the context of tumor growth. Consistent with previous reports, we observed that WT neutrophils exhibit suppressive functions toward T cell proliferation, survival, and activation in vitro 


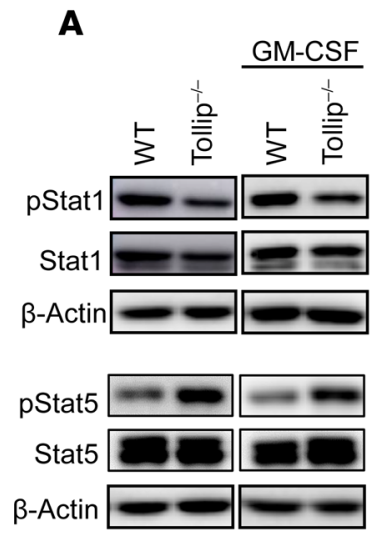

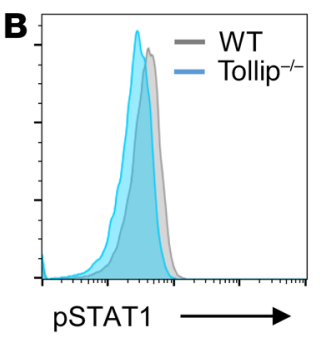

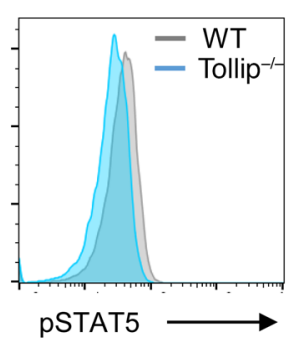

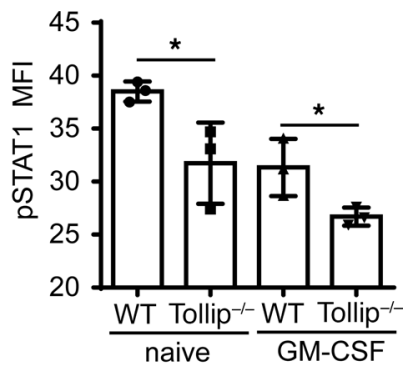

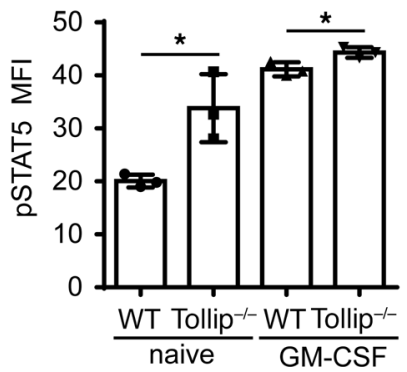

Figure 5. Tollip deficiency released the neutrophil suppression on T cell proliferation via PD-L1/CD80 signaling pathway. (A) Immunoblotting analysis of STAT1 and STAT5 in lysates from fresh bone marrow neutrophils or neutrophils primed with GM-CSF overnight. (B) Flow cytometry analysis of phospho-proteins in fresh bone marrow neutrophils or neutrophils primed with GM-CSF overnight, pregated on Ly6 $\mathrm{C}^{+}$cells. ${ }^{*} P<0.05$ by Student's $t$ test.

partly through the immune checkpoint PD-L1, resembling the tumor-promoting and $\mathrm{T}$ cell-suppressing effects of tumor-associated neutrophils in vivo (6). In contrast, we document in this report that Tollip ${ }^{-1-}$ neutrophils actually promote the survival and activation of $\mathrm{CD}^{+}$and $\mathrm{CD} 8^{+} \mathrm{T}$ cells. Recent studies reveal the significance of innate immune checkpoint molecules such as PD-L1 in suppressing $\mathrm{T}$ cell function and promoting tumor immune evasion (4). Our current study not only confirms the critical involvement of PD-L1 and CD80 on neutrophils in modulating T cell function, but also reveals that Tollip deficiency reprograms neutrophils into a T cell-promoting state with significantly reduced PD-L1 and elevated CD80. In addition to altered expression of CD80 and PD-L1, however, we can not rule out other molecular alterations due to Tollip deficiency in neutrophils that may also contribute to the enhanced $\mathrm{T}$ cell activation phenotype that we observed in this study. Emerging studies paint an extremely complex activation profile of neutrophils, with their ever-expanding roles in the modulation of diverse immune environments (30, 31), which clearly can not be fully addressed within any single study. Future studies that combine both transcriptomics as well as proteomics approaches are required to provide a complete picture of neutrophil activation dynamics related to cancer pathogenesis and/or treatment.

Our current study also provides molecular mechanisms responsible for the reprogramming of tumor-suppressing neutrophils. The expression of immune checkpoint T cell inhibitory molecule PD-L1 on neutrophils was shown to be under the control of STAT1 $(21,22)$. We observed that Tollip deficiency leads to reduced activation of STAT1 in Tollip ${ }^{-1}$ neutrophils that is consistent with reduced PD-L1 expression. On the other hand, STAT5 is responsible for the expression of costimulatory molecules such as CD80 on neutrophils (20). Our finding that Tollip-deficient neutrophils have elevated STAT5 activation provides a mechanistic explanation for the observed elevated CD80 expression. The molecular mechanisms responsible for the regulation of STAT1 and STAT5 by Tollip in neutrophils, however, remain to be better studied. Our recent biochemical study using cultured cell lines reveals that Tollip deletion may cause a disruption of lysosome-autophagosome fusion, leading to the stabilization and activation of STAT5 (32). On the other hand, Tollip may also be involved in the activation of the TBK complex at mitochondria, potentially explaining its activating role in STAT1 activation (33). These previous biochemical studies have laid a reasonable foundation for the functional role of Tollip in differentially modulating the activation of STAT1 and STAT5. Future detailed analyses are needed to better determine the molecular mechanisms responsible for the differential activation of STAT1 and STAT5 by Tollip in neutrophils.

From a translational perspective, our data reveal the potential for using reprogrammed innate leukocytes such as neutrophils in the intervention of CRC. Although improved diagnosis and preventive care 
A

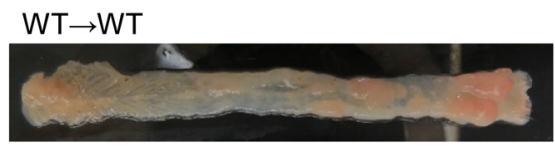

Tollip $^{-l-} \rightarrow$ WT

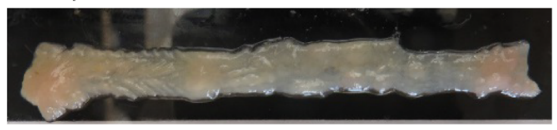

C

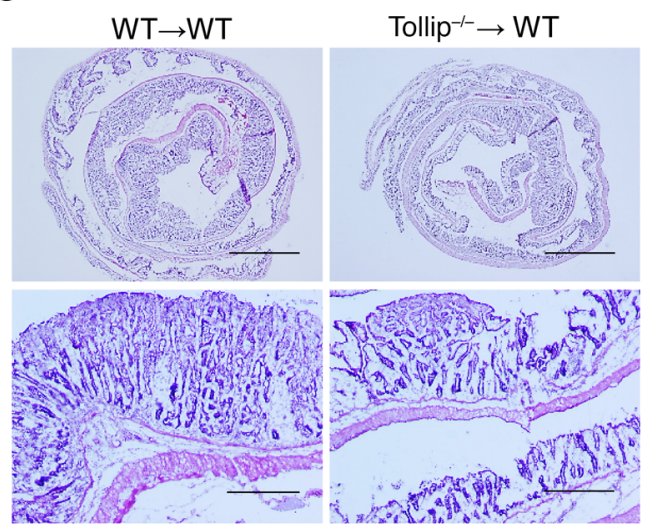

E
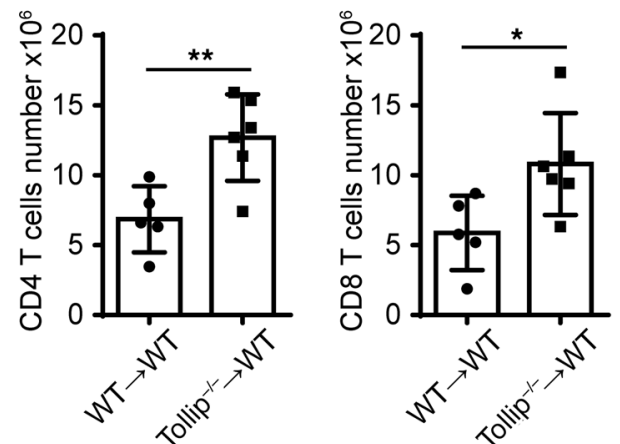

B

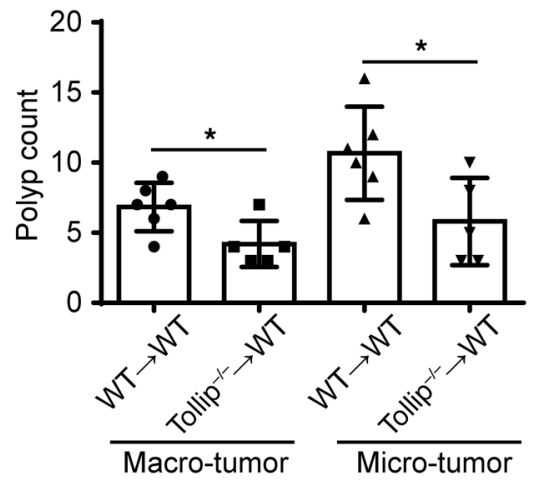

D

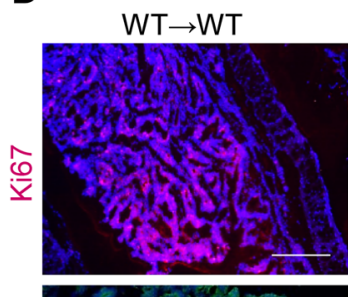

$$
\text { Tollip }^{-/} \rightarrow \mathrm{WT}
$$

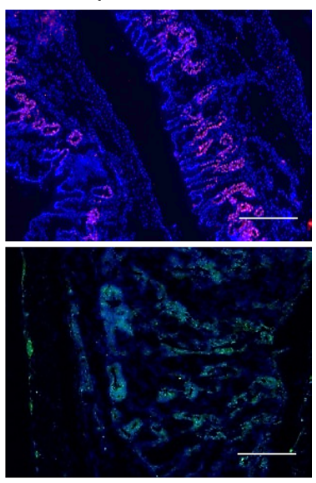

$\mathbf{F}$

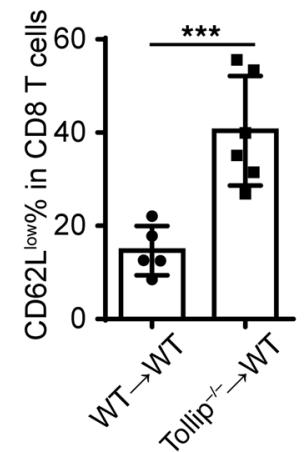

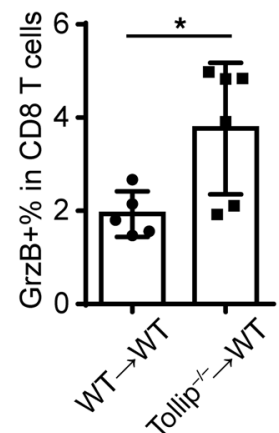

Figure 6. Adoptive transfer of Tollip ${ }^{-/-}$neutrophils to WT mice slows down colitis-associated cancer progression. (A) Representative images of colons on day 64 from WT mice that received WT or Tollip ${ }^{-1-}$ neutrophils. (B) Graphical representation of tumor burden in WT mice that received WT or Tollip ${ }^{-/-}$neutrophils. $n \geq 5$ per group. (C) H\&E-stained sections of colons from the mice that received WT or Tollip ${ }^{-1-}$ neutrophils. Colons were collected in Swiss rolls at the end of the AOM-DSS regimen. Scale bars: $2.5 \mathrm{~mm}$ (top) and $0.5 \mathrm{~mm}$ (bottom). (D) Immunofluorescence analysis of Ki67 and $\beta$-catenin. Blue color is DAPI staining. Scale bars: $200 \mu \mathrm{m}$. (E) CD4 ${ }^{+}$and CD8 ${ }^{+}$cell counts in the spleens from the mice that

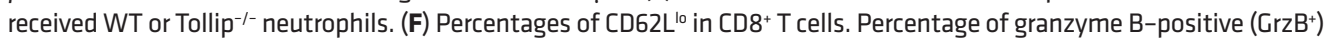
cells in CD8 ${ }^{+}$T cells. Statistical significance compared with WT in the same treatment conditions was determined by Student's $t$ test $(\mathbf{B})$ or Mann-Whitney $U$ test $(\mathbf{E}$ and $\mathbf{F}) .{ }^{*} P<0.05,{ }^{* *} P<0.01,{ }^{* * *} P<0.001$.

have led to a significant drop in CRC incidence in developed nations, CRC remains a lethal global disease and is projected to increase over $60 \%$ to more than 2 million new cases by 2030 worldwide (12). There has been a resurgence in cancer immune-therapy, given the intriguing yet limited success through engineered $\mathrm{T}$ cells combined with checkpoint inhibitors (2). Recent studies also suggest that subsets of neutrophils may hold potential in suppressing cancer cell growth, although underlying mechanisms for the differentiation/ activation of tumor-suppressing neutrophils are not clear (5). Our adoptive transfer data with Tollip-deficient neutrophils provide a proof of principle for the translational potential of reprogrammed neutrophils in the treatment of CRC. We realize that the pathogeneses of distinct cancer types may vary drastically and most 
likely involve distinct molecular as well as cellular mechanisms. Therefore, separate systematic and focused studies are necessary to address corresponding mechanisms and intervention strategies for each particular cancer type. Our current study is limited to the chemically induced CRC model, and does not intend to make a generalized conclusion for other cancer types. Nevertheless, our study provides an intriguing target in Tollip that may be tested in other tumor settings with context-dependent and pathologically relevant animal models in the future.

Collectively, our current study reveals that neutrophils engineered with Tollip deletion can be effectively reprogrammed into what we believe is a novel state to exhibit an effective antitumor immune defense. Mechanistically, we demonstrate that Tollip-deficient neutrophils can potently activate the functions of both CD4 ${ }^{+}$ and $\mathrm{CD}^{+} \mathrm{T}$ cells. Although we observed that the transfusion of Tollip-deficient monocytes fails to provide protection against AOM-DSS-induced CRC progression, however, we cannot rule out the possibility that reprogrammed neutrophils due to Tollip deletion may also affect other tumor-fighting immune cells such as NK cells or innate lymphocytes, which should be systematically examined in future studies. Mechanistic studies regarding molecular regulatory networks related to Tollip regulation are also needed in order to better characterize the therapeutic potential of Tollip-targeting drugs in designing precision cancer medicine.

\section{Methods}

Mice. WT C57BL/6 mice and Tollip ${ }^{-/-}$mice were bred and maintained in the animal facility at Virginia Tech in accordance with an IACUC-approved protocol. All littermate mice were 8-10 weeks of age and 25-30 g weight when experiments were initiated.

Experimental design. WT and Tollip ${ }^{-1-}$ mice received a single i.p. injection of AOM (Sigma-Aldrich) at a dose of $10 \mathrm{mg} / \mathrm{kg}$ body weight. A week after AOM injection, the mice were given 3 cycles of $2 \%$ DSS (MP Biomedicals) for 5 days followed by 14 days of normal drinking water. After the last water cycle mice were sacrificed and tissues were harvested for further analysis. A schematic of the protocol is shown in Figure 1A. Body weight, stool consistency, and bleeding were measured as part of the clinical score (score $0-4$, with a higher score corresponding to worse condition). Polyp formation was classified as "macro" or "micro" depending on the size equal to or greater than $2 \mathrm{~mm}$ versus less than $2 \mathrm{~mm}$, respectively. Independent experiments of AOM-DSS-induced colorectal tumorigenesis were conducted more than 3 times, and for every experiment there were at least 5 mice in each group.

Histology. Histological analyses of colon tissues were performed on freshly frozen OCT-embedded and sectioned slides $(5 \mu \mathrm{m})$. Slides were fixed in $4 \%$ neutral buffered formalin for 5 minutes, followed by H\&E staining.

Immunofluorescence. Immunofluorescence analyses were performed on freshly frozen OCT-embedded and sectioned slides $(5 \mu \mathrm{m})$. At least 6 mice from WT and Tollip ${ }^{-/}$mice were used for the study. For the measurement of Ki67 (Abcam, ab16667) and $\beta$-catenin (Cell Signaling Technology, 8814), sections were fixed in $4 \%$ neutral buffered formalin for 5 minutes, and stained with anti-mouse primary antibodies (1:100) followed by a biotinylated anti-Ig secondary Ab (BD Biosciences, 550338) and streptavidin-PE (eBioScience, 12-4317-87) or -FITC (BioLegend, 405202). DAPI was used to stain nuclei. Multiple viewing fields from each slide were captured under a fluorescence microscope. Pixel values reflecting the fluorescence intensities of each viewing field were quantified with NIH ImageJ software.

Immunoblotting. Bone marrow neutrophils were purified by $65 \%$ Percoll gradient and the purity was greater than $90 \%$, as confirmed by $\mathrm{Ly} 6 \mathrm{G}^{+} \mathrm{CD} 11 \mathrm{~b}^{+}$staining. For neutrophil culture, purified neutrophils were cultured in RPMI 1640 medium supplemented with 10\% fetal bovine serum (FBS), 2 mM L-glutamine, $10 \mathrm{mM}$ HEPES, 1\% penicillin/streptomycin, and $1 \mathrm{ng} / \mathrm{ml}$ GM-CSF overnight. Naive or cultured neutrophils were harvested in SDS lysis buffer containing protease and phosphatase inhibitors as previously described (34). Briefly, equal amounts of protein were applied to SDS-PAGE and transferred to PVDF membranes (Bio-Rad). The membranes were blocked with 5\% nonfat dry milk, and then incubated with primary anti-phospho-STAT1 (Cell Signaling Technology, 9177), anti-STAT1 (Santa Cruz Biotechnology, SC-346), anti-STAT5 (Cell Signaling Technology, 94205), anti-phospho-STAT5 (Cell Signaling Technology, 9359), or $\beta$-actin antibody (Santa Cruz Biotechnology, SC-47778), and anti-rabbit or mouse IgG secondary antibody (Cell Signaling Technology) according to the manufacturer's instructions. The immunoblots were developed by a chemiluminescence ECL detection kit (Thermo Fisher Scientific).

Adoptive transfer of neutrophils. Bone marrow neutrophils from donor mice (WT or Tollip ${ }^{-/}$) were purified ( $>90 \%$ confirmed by flow cytometry) using an EasySep Mouse Neutrophil Enrichment Kit (STEMCELL Technologies), according to the manufacturer's instructions. Recipient WT mice were 
transfused twice (after DSS day 5 and day 12) per DSS-resting cycle through i.v. injection with $2.4 \times 10^{6}$ WT or Tollip ${ }^{-1-}$ neutrophils suspended in $200 \mu$ sterile PBS. A detailed timeline is illustrated in Supplemental Figure 6A.

Adoptive transfer of monocytes. Bone marrow cells isolated from WT or Tollip ${ }^{-1-}$ mice were cultured in RPMI 1640 medium supplemented with 10\% FBS, 2 mM L-glutamine, 10 mM HEPES, 1\% penicillin/ streptomycin, and $10 \mathrm{ng} / \mathrm{ml} \mathrm{M}-\mathrm{CSF}$ for 5 days. Recipient WT mice were transfused twice per cycle through i.v. injection with $3 \times 10^{6} \mathrm{WT}_{\text {or Tollip }}{ }^{-/}$monocytes suspended in $200 \mu 1$ sterile PBS. A detailed timeline is illustrated in Supplemental Figure 10A.

ELISA. The levels of IL-1 $\beta$, TGF- $\beta$, IFN- $\gamma$, and IL-12 were measured using ELISA kits purchased from $\mathrm{R} \& \mathrm{D}$ Systems, according to the manufacturer's instructions.

Lamina propria cell isolation. Colons were opened longitudinally and cleaned by flushing with ice-cold PBS. A single-cell suspension was prepared using Lamina Propria dissociation Kit (MACS). Briefly, the colons were cut into pieces, and incubated with HBSS containing $5 \mathrm{mM}$ EDTA, 5\% FBS, and $1 \mathrm{mM}$ DTT to remove epithelial cells. The remaining pieces were then incubated with HBSS containing 5\% FBS and enzyme mix using the gentleMACS dissociator. The cells were washed, passed through a 70- $\mu \mathrm{m}$ strainer, and resuspended in FACS buffer for further flow cytometry analyses.

Flow cytometry. Fluorescent-conjugated anti-mouse antibodies specific for PD-L1 (clone 10F.9G2), CD80 (clone 16-10A1), CD14 (clone M14-23), CD11b (clone M1/70), CD4 (clone GK1.5), CD8 (clone 53-6.7), Ly6G (clone 1A8), and CD45 (clone 30-F11) were purchased from BioLegend. PI was also added to determine the cell viability. Peripheral blood cells and splenocytes were harvested from WT and Tollip ${ }^{-/-}$mice as previously described (35). The cells were washed in FACS buffer (HBSS supplemented with $2 \%$ FBS and $0.02 \%$ sodium azide) and stained with fluorescently labeled antibodies for 20 minutes on ice. Stained cells were analyzed with a FACSCanto II (BD Biosciences). FACS plots shown were analyzed with FlowJo software.

$T$ cell proliferation assay. Splenocytes were labeled with CFSE (5,6-carboxyfluorescein diacetate succinimidyl ester) (Invitrogen, Molecular Probes), according to the manufacturer's instructions. CFSE-labeled splenocytes were stimulated with plate-bound anti-mouse CD3 antibody (eBioscience, clone 145-2C11). Neutrophils purified from bone marrow were cultured in RPMI 1640 medium supplemented with 10\% FBS, $2 \mathrm{mM}$ L-glutamine, $10 \mathrm{mM}$ HEPES, $1 \%$ penicillin/streptomycin, and 1 $\mathrm{ng} / \mathrm{ml}$ GM-CSF for 24 hours. CFSE-labeled splenocytes were mixed with cultured neutrophils at a 1:1 ratio and cocultured in anti-CD3-coated plates for 72 hours. CFSE signals were analyzed by flow cytometry on gated $\mathrm{CD}^{+}$and $\mathrm{CD} 8^{+}$cells. In blocking experiments, antibodies against CD 80 or PD-L1 (BioLegend) were add to the coculture at the concentration of $1 \mu \mathrm{g} / \mathrm{ml}$. To test the viability of $\mathrm{T}$ cells after coculture, neutrophils and $\mathrm{T}$ cells were treated as above but plated in 96-well U-bottom plates without anti-CD3 coating.

Statistics. All experiments were performed at least 3 times. Representative and reproducible results were utilized for this study. Statistical analysis was performed with GraphPad Prism software (version 6.0). Values are expressed as mean \pm SD. Unpaired 2-tailed Student's $t$ test, Welch's test, Mann-Whitney $U$ test, or 1-way ANOVA (followed by Tukey's post hoc comparisons) were used to analyze differences between groups, as appropriate. A $P$ value $<0.05$ was considered statistically significant.

Study approval. All experiments were performed in accordance with protocols approved by the IACUC at Virginia Tech (protocol number BIO-17-193).

\section{Author contributions}

LL and YZ conceived the project, designed the studies, proposed the experiments, and wrote the manuscript. YZ, CL, and SG performed the experiments.

\section{Acknowledgments}

We appreciate the help of Li Lab members for assistance, and Allison Rahtes for critical proofreading. This study was supported in part by grants from NIH R01 AI136386.

Address correspondence to: Liwu Li, 970 Washington Street, Virginia Tech, Blacksburg, Virginia 24061-0910, USA. Phone: 540.231.1433; Email: 1wli@vt.edu. 
1. Binnewies M, et al. Understanding the tumor immune microenvironment (TIME) for effective therapy. Nat Med. 2018;24(5):541-550.

2. Lim WA, June CH. The principles of engineering immune cells to treat cancer. Cell. 2017;168(4):724-740.

3. Fletcher R, Wang YJ, Schoen RE, Finn OJ, Yu J, Zhang L. Colorectal cancer prevention: Immune modulation taking the stage. Biochim Biophys Acta Rev Cancer. 2018;1869(2):138-148.

4. Smyth MJ, Ngiow SF, Ribas A, Teng MW. Combination cancer immunotherapies tailored to the tumour microenvironment Nat Rev Clin Oncol. 2016;13(3):143-158.

5. Coffelt SB, Wellenstein MD, de Visser KE. Neutrophils in cancer: neutral no more. Nat Rev Cancer. 2016;16(7):431-446.

6. Wang TT, et al. Tumour-activated neutrophils in gastric cancer foster immune suppression and disease progression through GM-CSF-PD-L1 pathway. Gut. 2017;66(11):1900-1911.

7. Nayak A, McDowell DT, Kellie SJ, Karpelowsky J. Elevated preoperative neutrophil-lymphocyte ratio is predictive of a poorer prognosis for pediatric patients with solid tumors. Ann Surg Oncol. 2017;24(11):3456-3462.

8. Powell DR, Huttenlocher A. Neutrophils in the tumor microenvironment. Trends Immunol. 2016;37(1):41-52.

9. Burns K, et al. Tollip, a new component of the IL-1RI pathway, links IRAK to the IL-1 receptor. Nat Cell Biol. 2000;2(6):346-351.

10. Lu K, Psakhye I, Jentsch S. Autophagic clearance of polyQ proteins mediated by ubiquitin-Atg8 adaptors of the conserved CUET protein family. Cell. 2014;158(3):549-563.

11. Chen K, Yuan R, Zhang Y, Geng S, Li L. Tollip deficiency alters atherosclerosis and steatosis by disrupting lipophagy. $J$ Am Heart Assoc. 2017;6(4):e004078.

12. Arnold M, Sierra MS, Laversanne M, Soerjomataram I, Jemal A, Bray F. Global patterns and trends in colorectal cancer incidence and mortality. Gut. 2017;66(4):683-691.

13. Patel SG, Ahnen DJ. Colorectal cancer in the young. Curr Gastroenterol Rep. 2018;20(4):15.

14. Diao N, et al. Deficiency in Toll-interacting protein (Tollip) skews inflamed yet incompetent innate leukocytes in vivo during DSS-induced septic colitis. Sci Rep. 2016;6:34672.

15. De Robertis M, et al. The AOM/DSS murine model for the study of colon carcinogenesis: From pathways to diagnosis and therapy studies. J Carcinog. 2011;10:9.

16. Hu B, et al. Inflammation-induced tumorigenesis in the colon is regulated by caspase-1 and NLRC4. Proc Natl Acad Sci USA 2010;107(50):21635-21640.

17. Thaker AI, et al. IDO1 metabolites activate $\beta$-catenin signaling to promote cancer cell proliferation and colon tumorigenesis in mice. Gastroenterology. 2013;145(2):416-25.e1.

18. Kim SV, et al. GPR15-mediated homing controls immune homeostasis in the large intestine mucosa. Science. 2013;340(6139):1456-1459.

19. Gargett T, et al. GM-CSF signalling blockade and chemotherapeutic agents act in concert to inhibit the function of myeloid-derived suppressor cells in vitro. Clin Transl Immunology. 2016;5(12):e119.

20. Tormo AJ, Gauchat JF. A novel role for STAT5 in DC: Controlling the Th2-response. JAKSTAT. 2013;2(4):e25352.

21. O'Reilly LA, et al. Loss of NF- $\mathrm{BB} 1$ causes gastric cancer with aberrant inflammation and expression of immune checkpoint regulators in a STAT-1-dependent manner. Immunity. 2018;48(3):570-583.e8.

22. Garcia-Diaz A, et al. Interferon receptor signaling pathways regulating PD-L1 and PD-L2 expression. Cell Rep. 2017;19(6):1189-1201.

23. Awad RM, De Vlaeminck Y, Maebe J, Goyvaerts C, Breckpot K. Turn back the TIMe: Targeting tumor infiltrating myeloid cells to revert cancer progression. Front Immunol. 2018;9:1977.

24. Houghton AM. The paradox of tumor-associated neutrophils: fueling tumor growth with cytotoxic substances. Cell Cycle. 2010;9(9):1732-1737.

25. Kiss M, Van Gassen S, Movahedi K, Saeys Y, Laoui D. Myeloid cell heterogeneity in cancer: not a single cell alike. Cell Immunol. 2018;330:188-201.

26. Ben-Meir K, Twaik N, Baniyash M. Plasticity and biological diversity of myeloid derived suppressor cells. Curr Opin Immunol. 2018;51:154-161

27. Shaul ME, Fridlender ZG. Neutrophils as active regulators of the immune system in the tumor microenvironment. J Leukoc Biol. 2017;102(2):343-349.

28. Shaul ME, et al. Tumor-associated neutrophils display a distinct N1 profile following TGF $\beta$ modulation: A transcriptomics analysis of pro- vs. antitumor TANs. Oncoimmunology. 2016;5(11):e1232221.

29. Sionov RV, Fridlender ZG, Granot Z. The multifaceted roles neutrophils play in the tumor microenvironment. Cancer Microenviron. 2015;8(3):125-158.

30. Casanova-Acebes M, et al. Neutrophils instruct homeostatic and pathological states in naive tissues. J Exp Med. 2018;215(11):2778-2795.

31. Ran T, Geng S, Li L. Neutrophil programming dynamics and its disease relevance. Sci China Life Sci. 2017;60(11):1168-1177.

32. Kowalski E, Geng S, Rathes A, Lu R, Li L. Toll-interacting protein differentially modulates HIF1 $\alpha$ and STAT5-mediated genes in fibroblasts. J Biol Chem. 2018;293(31):12239-12247.

33. Maitra U, et al. Molecular mechanisms responsible for the selective and low-grade induction of proinflammatory mediators in murine macrophages by lipopolysaccharide. J Immunol. 2012;189(2):1014-1023.

34. Chen K, Geng S, Yuan R, Diao N, Upchurch Z, Li L. Super-low dose endotoxin pre-conditioning exacerbates sepsis mortality EBioMedicine. 2015;2(4):324-333.

35. Geng S, et al. The persistence of low-grade inflammatory monocytes contributes to aggravated atherosclerosis. Nat Commun. $2016 ; 7: 13436$ 